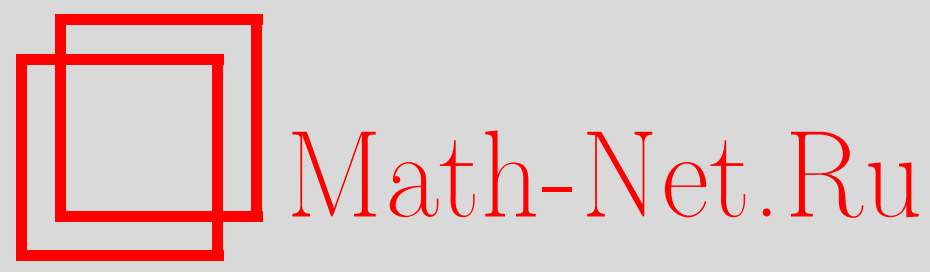

А. С. Демидов, Ж. Мвамбакана, И. А. Федотов, Альтернирующий метод Шварца для эллиптических краевых задач, удовлетворяющих принципу максимума, Матем. заметки, 2005, том 78, выпуск 4, 622-624

DOI: https://doi.org/10.4213/mzm2622

Использование Общероссийского математического портала Math-Net.Ru подразумевает, что вы прочитали и согласны с пользовательским соглашением http://www.mathnet.ru/rus/agreement

Параметры загрузки:

IP : 52.87 .193 .239

26 апреля 2023 г., 16:38:47

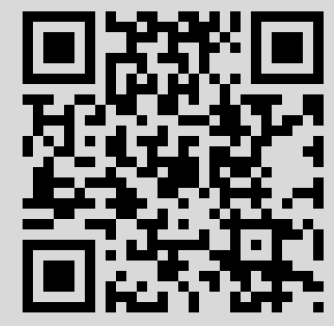




\section{АЛЬТЕРНИРУЮЩИЙ МЕТОД ШВАРЦА ДЛЯ ЭЛЛИПТИЧЕСКИХ КРАЕВЫХ ЗАДАЧ, УДОВЛЕТВОРЯЮЩИХ ПРИНЦИПУ МАКСИМУМА}

\section{А. С. Демидов, Ж. Мвамбакана, И. А. Федотов}

Рассматривается построение решения эллиптической краевой задачи

$$
\begin{gathered}
\left.A u\right|_{x \in \omega} \stackrel{\text { def }}{=} \sum_{i, j=1}^{n} a_{i j}(x) \frac{\partial^{2} u(x)}{\partial x_{i} \partial x_{j}}+\sum_{k=1}^{n} b_{k}(x) \frac{\partial u(x)}{\partial x_{k}}+c(x) u(x)=f(x), \\
\left.\left.B u\right|_{s \in \partial_{r} \omega} \stackrel{\text { def }}{=} \alpha(s) \frac{\partial u}{\partial \nu}\right|_{s \in \partial_{r} \omega}+\beta(s) u(s)=g(s)
\end{gathered}
$$

для "сложных" областей $\omega \Subset \mathbb{R}^{n}$, т.е. таких, которые представимы в виде объединения конечного числа "простых" областей $\omega_{k}$. При этом "простые" области - это те, для которых задачи вида (1), (2) (с заменой $\omega$ на $\omega_{k}$ ) могут быть “легко" построены. Мы ограничимся рассмотрением таких "простых" областей, которые могут быть представлены как пересечение конечного числа $n$-мерных областей с гладкой $(n-1)$-мерной границей. При этом предполагается, что в точках пересечения гладких $(n-1)$-мерных частей границы (как $\partial \omega$, так и $\left.\partial \omega_{k}\right)$ нормали к этим частям границы не коллинеарны. В качестве "простых" областей могут быть, например, рассмотрены

1) ограниченная $n$-мерная область с гладкой $(n-1)$-мерной границей, и

2) параллелепипед (возможно, “криволинейный”).

Пример “сложной” области представлен на рис. 1.
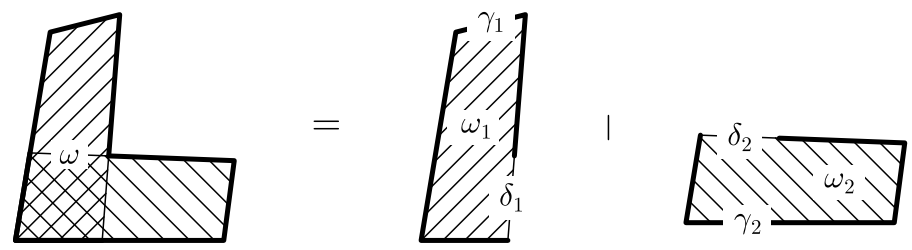

Рис. 1. Область $\omega \subset \mathbb{R}^{n}$ с границей $\gamma=\partial \omega$, составленная из "параллелепипедов" $\omega_{1}$ и $\omega_{2}$; при этом $\partial \omega_{k}=\gamma_{k} \cup \delta_{k}$, где $\delta_{k}=\partial \omega_{k} \cap \omega_{3-k}, k=1,2$

Объединение гладких $(n-1)$-мерных частей границы области $\omega$ будем обозначать $\partial_{r} \omega$. Сингулярную часть границы, т.е. $\partial \omega \backslash \partial_{r} \omega$, обозначим $\partial_{s} \omega$.

Под решением задачи (1), (2) понимается функция $u: \omega \ni x \mapsto u(x)$, которая

1) непрерьвна и ограничена на $\bar{\omega} \backslash \partial_{s} \omega$;

2) имеет ограниченные предельные значения в граничных точках $\partial_{r} \omega_{k}$;

3) удовлетворяет всем условиям задачи (1), (2), включая существование соответствующих производных.

Предполагается, что функции $a_{i j}, b_{k}, c$ и $f$, фигурирующие в (1), непрерывны в $\bar{\omega}$ и при $x \in \bar{\omega}$ выполнены условия

$$
c(x) \leqslant 0, \quad \sum_{i, j=1}^{n} a_{i j}(x) \xi_{i} \xi_{j} \geqslant a_{0}|\xi|^{2}, \quad \text { где } \xi=\left(\xi_{1}, \ldots, \xi_{n}\right) \in \mathbb{R}^{n}, a_{0}>0 .
$$

Что касается функций $\alpha, \beta$ и $g$, фигурирующих в (2), то предполагается, что они непрерьвны вплоть до границы каждой компоненты связности регулярной части $\partial_{r} \omega$ границы области $\omega$, причем

$$
\alpha(s) \beta(s) \geqslant 0, \quad \inf _{s}[\alpha(s)+\beta(s)]>0, \quad \beta(s) \not \equiv 0 \quad \text { при } s \in \partial_{r} \omega .
$$

Работа выполнена при поддержке Российского фонда фундаментальных исследований, грант № 04-01-00735. 
В 1869 г. Шварц предложил идею построения решения задачи Дирихле для 2-мерного уравнения Лапласа в "сложной" области $\omega=\bigcup_{k=1}^{m} \omega_{k}$ с помощью итерационного процесса (см., например, [1], [2] или [3]), в котором последовательно фигурируют решения задач Дирихле в "простых" областях $\omega_{k}$. Алгоритмы такого рода принято называть альтернирующим методом Швариа. Отметим, что близкая идея была использована в [4] и [5] для построения решения эллиптических краевых задач с нелинейньми условиями сопряжения.

В данной работе мы применяем идею Шварца к задаче (1), (2), имея в виду построение такого алгоритма численного решения, который был бы эффективен при анализе влияния на искомое решение функциональных параметров $\alpha$ и $\beta$, входящих в граничное условие (2). Такой анализ предполагает численное решение большой серии однотипных краевых задач. В этой ситуации есть резон попытаться построить функцию Грина. Это сравнительно легко сделать для некоторых "простых" областей (по крайней мере, в случае оператора Лапласа). Альтернирующий метод Шварца позволяет "обыпрать" это обстоятельство и получить более эффективное средство исследования по сравнению с изучением серии задач непосредственно в исходной "сложной" области.

Чтобы не перегружать изложение, рассмотрим здесь лишь один конкретный случай, представляющий наиболее сложную ситуацию: ту, когда пересекаются границы "простых" областей ${ }^{1}$. Именно такая ситуация возникает в задаче, инициировавшей данную работу ${ }^{2}$. Поэтому мы ограничимся здесь случаем $L$-образной области $\omega$, схематически представленной на рисунке.

Теорема. Решение и задачи (1), (2) существует, однозначно определено на $\bar{\omega} \backslash \partial_{s} \omega$. При этом,

$$
\left\|u-u_{k j}\right\|_{\omega_{k}} \stackrel{\text { def }}{=} \max _{x \in \omega_{k}}\left|u(x)-u_{k j}(x)\right| \rightarrow 0 \quad \text { npu } \quad 1 \leqslant j \rightarrow \infty
$$

где $u_{1 j}$ и $u_{2 j}$ - решения следующих задач в "простых" областях $\omega_{1} u \omega_{2}$ :

$$
A u_{k j}=f \quad \text { в } \quad \omega_{k}, \quad B u_{k j}=g \quad \text { на } \quad \gamma_{k}, \quad u_{k j}=g_{k j} \quad \text { на } \quad \delta_{k} .
$$

Здесь непрерывная на $\overline{\delta_{1}}$ функция $g_{11}$ мохет быть выбрана произвольно (мохсно счиmamb, чmo $\left.g_{11}=0\right)$, a $g_{1 j}=\left.u_{2, j-1}\right|_{\delta_{1}}, g_{2 j}=\left.u_{1 j}\right|_{\delta_{2}}$.

ДокАЗАТЕльство. Зафииксируем $M \geqslant 1$ и положим $v_{k j}=u_{k, j+M}-u_{k j}$. Имеем

$$
A v_{k j}=0 \text { в } \omega_{k}, \quad B v_{k j}=0 \text { на } \gamma_{k}, \quad v_{k j}=\varphi_{k j} \text { на } \delta_{k},
$$

где $\varphi_{1 j}=\left.v_{2, j-1}\right|_{\delta_{1}}, \varphi_{2 j}=\left.v_{1 j}\right|_{\delta_{2}}$. В силу условий (3) для функции $v_{k}$ (мы опускаем здесь у $v_{k j}$ второй индекс) справедлива GHO-лемма о нормальной производной (доказанная Жиро [9] в плоском случае, а затем в 1952 году независимо Хопфом [10] и Олейник [11] для любой размерности). Согласно этой лемме, максимум модуля функции $v_{k}$ достигается на границе области $\omega_{k}$, причем в точке $s \in \partial \omega_{k}$, где достигается этот максимум, нормальная производная ${ }^{3} \partial v_{k} / \partial \nu$ отлична от нуля (если, конечно, $\left.v_{k} \not \equiv 0\right)$. Проверим, что максимум модуля функции $v_{k}$ достигается на $\delta_{k}=\partial \omega_{k} \cap \omega_{3-k}$ (см. рис. 1$)$. В силу GHO-леммы, этот максимум не может достигаться на той части $\gamma_{k}=\partial \omega_{k} \backslash \delta_{k}$, где коэффициент $\beta$, фигурирующий в формуле $(2)$, равен нулю. Да и на той части $\gamma_{k}$, где $\beta \neq 0$, максимум модуля функции $v_{k}$ тоже не может достигаться, ибо иначе

\footnotetext{
${ }^{1}$ Случай, когда "простые" области $\omega_{k}$ пересекаются без пересечения своих границ рассматривался в [6] при построении решения внешней краевой задачи для уравнения Лапласа. При этом брались две “простые” области: 1) внешность шара радиуса $r$ (там явно вьписывается решение, зависящее только от радиуса) и 2) пересечение исходной области с шаром радиуса $R>r$ (там решение строится численно). Такого сорта построение было реализовано в [7] и в некоторых случаях для уравнения Гельмголца при условиях Зоммерфельда на бесконечности.

${ }^{2}$ Речь идет о задаче [8], связанной с вопросом спектрального анализа исследуемого вещества, помещенного в графитовую печь. Требование чистоты эксперимента необходимо влечет "сложную" форму этой печи.

ЗЭто верно и для нормальной производной к каждой из гладких частей границы в точках их пересечения.
} 
внутри $\omega_{k}$ нашлась бы точка ${ }^{4} \tilde{s}$, в которой величина $\left|v_{k}(\tilde{s})\right|$ была бы болше, чем предполагаемый $\max \left|v_{k}\right|=\left|v_{k}(\hat{s})\right|$ (на той части $\gamma_{k}$, где $\beta \neq 0$ ). Итак,

$$
\max _{\overline{\omega_{k}}}\left|v_{k}\right| \leqslant \max _{\delta_{k}}\left|v_{k}\right| \text {. }
$$

Ниже мы покажем, что

$$
\max _{\delta_{k}}\left|v_{k}\right| \rightarrow 0 \quad \text { при } j \rightarrow \infty .
$$

Тем самьм, будет доказана теорема, поскольку (5) следует из (8) и (9), а единственность на $\bar{\omega} \backslash \partial_{s} \omega$ вытекает из GHO-леммы.

Свойство (9) есть следствие того, что существует константа $q<1$ такая, что

$$
\max _{\delta_{k}}\left|v_{k, j+1}\right| \leqslant q \max _{\delta_{k}}\left|v_{k j}\right| .
$$

Для доказательства неравенства (10) рассмотрим оператор

$$
G_{k l}:\left.C\left(\delta_{k}\right) \ni \varphi \mapsto v_{\varphi}\right|_{\delta_{l}} \in C\left(\delta_{l}\right), \quad k \neq l .
$$

Здесь через $v_{\varphi}$ обозначено решение задачи (7) в области $\omega_{k}\left(\right.$ с заменой $\varphi_{k j}$ на $\varphi$ и $v_{k j}$ на $\left.v_{\varphi}\right)$, а $C(\delta)$ - это пространство непрерывных функций на $\delta$, продолжающихся по непрерывности на $\bar{\delta}$. Это пространство снабжено нормой

$$
\|\varphi\|_{\delta}=\max _{s \in \delta}|\varphi(s)| .
$$

В силу теоремы Арцела оператор $G_{k l}$ компактен, поскольку функция $\left.v_{\varphi}\right|_{\delta_{l}}$ дифференцируема вплоть до границы $\delta_{l}$. Действительно, $v_{\varphi}$ дважды непрерьвно дифференцируема в $\omega_{k}$ (и потому и на $\delta_{l}$ ), а в точках $\overline{\delta_{l}} \backslash \delta_{l}$ функция $v_{\varphi}$ дифференцируема, поскольку $B v_{\varphi}=0$. Заметим теперь, что

$$
v_{k, j+1}\left|\delta_{k}=G_{k} v_{k j}\right|_{\delta_{k}}, \quad \text { где } G_{k}=G_{l k} \circ G_{k l} \text {. }
$$

Для завершения доказательства неравенства (10), а потому и теоремы, нам достаточно проверить, что $\left\|G_{k}\right\|<1$, где

$$
\left\|G_{k}\right\|=\sup _{\|\varphi\|_{\delta} \leqslant 1}\left\|G_{k} \varphi\right\|_{\delta}, \quad \text { a } \delta=\delta_{k} .
$$

Пусть $\left\|\varphi_{j}\right\|_{\delta} \leqslant 1$ и $\left\|G_{l k} \psi_{j}\right\|_{\delta} \rightarrow \sup$, где $\psi_{j}=G_{k l} \varphi_{j}$. В силу компактности оператора $G_{k l}$ можно считать, что $\psi_{j} \rightarrow \hat{\psi}$ в $C\left(\delta_{l}\right)$. Непрерьвность оператора $G_{l k}$ влечет соотношение $\left\|G_{k}\right\|=$ $\max _{\delta_{k}}\left|G_{l k} \hat{\psi}\right|$. Остается заметить, что (в силу GHO-леммы) $\max _{\delta_{l}}|\hat{\psi}| \leqslant 1$ и, кроме того,

$$
\max _{\delta_{k}}\left|G_{l k} \hat{\psi}\right| \leqslant q \max _{\delta_{l}}|\hat{\psi}|, \quad \text { где } q<1 .
$$

Теорема полностью доказана.

\section{СПИСОК ЦИТИРОВАННОЙ ЛИТЕРАТУРЫ}

1. Курант Р. Уравнения с частными производными. М.: Мир, 1964. 2. Годунов С. К. Уравнения математической физики. М.: Наука, 1971. 3. Канторович Л. В., Крылов В. И. Приближенные методы высшего анализа (3-е изд.). М.-Л.: ГИТТЛ, 1950. 4. Демидов А. С. // УМН. 1994. T. 49. № 4. C. 127. 5. Demidov A. S., Yatchenko E. S. // Intern. J. Heat Mass Transfer. 1994. V. 37. № 14. P. 2155-2163. 6. Демидов А. С., Никифоров Е. А., Шубин М. А. Отчет ОКБ МЭИ и мех.-мат. фак-та МГУ. М., 1995. 7. Воскобойникова О. И. // Препринт ИПМ № 38. М.: ИПМ РАН им. М. В. Келдьша, 1997. 8. Katskov A. D., Marais P. J. J., Ngobeni P. // Spectrochimica Acta. 1998. V. 53B. P. 671-682. 9. Giraud G. // Bull. Sci. Math. 1932. V. 56. P. 248-272. 10. Hopf E. // Proc. Amer. Math. Soc. 1952. V. 3. P. 701-793. 11. Олейник О. А. // Матем. сб. 1952. Т. 30. № 3. С. 696-697.

(А. С. Демидов) Московский государственный университет им. М.В. Ломоносова

Поступило (А. С. Демидов, Ж. Мвамбакана, И. А. Федотов)

Tschwane University of Technology, г. Претория, ЮАР

E-mail: asd@math.msu.su, mwambakanajn@tut.as.za, fedotovi@tut.as.za

\footnotetext{
${ }^{4}$ В качестве $\tilde{s} \in \omega_{k}$ можно было бы взять любую точку, лежащую вблизи точки $\hat{s} \in \gamma_{k}$ в направлении внутренней нормали от $\hat{s} \in \gamma_{k}$. Это следует из условия (4), так как знак нормальной производной $\partial v_{k} / \partial \nu$ противоположен знаку $v_{k}$ в точках, где $\beta \neq 0$ (и $v_{k} \neq 0$ ).
} 